\title{
$\Lambda$ 有界变差函数的一些性质
}

\author{
王 斯 雷 \\ (杭 州大 学)
}

本文分三节, 第一节回答 Waterman 于 1976 年提出的一个问题; 第二节讨论 $\Lambda$ 有界变差 函数富里埃系数的阶; 第三节研究 $\Lambda$ 有界变差函数富里埃级数的绝对收敛问题. 所得结果分 别改进了 Waterman 相应的工作.

设 $f(x)$ 是定义在 $[a, b]$ 上的实函数, $\left\{I_{n}\right\}$ 是一列互不重叠的区间: $I_{n}=\left[a_{n}, b_{n}\right] \subset l=$ $[a, b]$, 并写 $f\left(I_{n}\right)=f\left(b_{n}\right)-f\left(a_{n}\right)$. 又设 $\Lambda$ 表示非降的正数列: $\boldsymbol{\Lambda}=\left\{\lambda_{n}\right\}$, 而且级数 $\sum_{1}^{\infty} 1 / \lambda_{n}$ 发散.

定义 $1 I=[a, b]$ 上定义的函数 $f(x)$, 如果对于任一列互不重叠的区间 $\left\{I_{n}\right\}, I_{n} \subset$ 1 , 级数

$$
\sum_{n=1}^{\infty}\left|f\left(I_{n}\right)\right| / \lambda_{n}<\infty,
$$

那么称 $f$ 是 $I$ 上的 $\Lambda$ 有界变差函数, 记 $f \in \Lambda B V$, 或 $f \in\left\{\lambda_{n}\right\} B V$. 特别是, 当 $\Lambda=\{n\}$ 时, 称 $f$ 为调和有界变差函数,记为 $f \in H B V$ 数

$$
V(I)=V_{A}(f ; I)=\sup \left\{\sum\left|f\left(I_{n}\right)\right| / \lambda_{n}: I_{n} \subset I\right\},
$$

称为 $f$ 在 $I$ 上的 $\Lambda$ 全变差.

定义 2 设 $f \in \Lambda B V$ ，记 $\Lambda_{m}=\left\{\lambda_{n+n}\right\}, m=1,2, \cdots$. 如

$$
V_{A_{m}}(f ; l) \rightarrow 0 \quad(m \rightarrow \infty),
$$

那么称 $f$ 在 $\Lambda$ 变差下是连续的, 并记 $f \in \Lambda B V_{c}$.

显然, 当 $\Lambda=\{1\}$, 即 $\Lambda B V$ 成为通常的有界变差函数类 $B V$ 时, 满足定义 2 的函数只 能是常数. 所以,考虑 $\Lambda$ 变差下连续的函数时, 应当假定 $\lambda_{n} \uparrow \infty$.

Waterman 在文献 [1]中提出如下的问题: 怎样可以刻划出在 $\Lambda$ 变差下是连续的函数? 就 是说, $f \in \triangle B V_{c}$ 的充分必要条件是什么?

我们回答如下:

定理 $1 f \in \Lambda B V_{c}$ 的充要条件是, 存在一列非降的正数列 $\Lambda^{1)}=\left\{\lambda_{n}^{(1)}\right\}, \lambda_{n}^{(1)} \uparrow \infty$, $\lambda_{n}^{(1)}=o\left(\lambda_{n}\right)(n \rightarrow \infty)$, 使 $f \in \Lambda^{(1)} B V$, 即

$$
\Lambda B V_{c}=\bigcup_{\Delta^{(1)}=\alpha(\Delta)} \Lambda^{(1)} B V,
$$

这里记号 $\Lambda^{(1)}=o(\Lambda)$ 表示数列 $\Lambda^{(1)}=\left\{\lambda_{n}^{(1)}\right\}$ 满足条件

本文1980年6月19日收到. 


$$
\lambda_{*}^{(1)}=o\left(\lambda_{n}\right) \quad(n \rightarrow \infty),
$$

而 (1.4) 式右方表示关于满足条件 $\Lambda^{(1)}=o(\Lambda)$ 的一切 $\Lambda^{(1)} B V$ 类作并集.

$$
=
$$

从现在起, 假设 $f$ 是周期为 $2 \pi$ 的周期函数， $I=[0,2 \pi]$, 并且在 $I$ 上, $f \in \Lambda B V$. Waterman 最近在文献 $[2]$ 中研究了 $f$ 的富里埃系数的阶的大小, 他的结果是这样的.

定理 A 若 $f \in \Lambda B V, \Lambda=\left\{\lambda_{n}\right\}$, 则 $f$ 的富里埃系数

$$
\begin{aligned}
& a_{n}(f) \\
& b_{n}(f)
\end{aligned}=\frac{1}{\pi} \int_{0}^{2 \pi} f(t) \frac{\cos n t}{\sin n t} d t=O\left(\frac{\lambda_{n}}{n}\right) \quad(n \rightarrow \infty) .
$$

我们指出, 当 $\lambda_{n}=n(n=1,2, \cdots)$ 时，(2.1) 式右方为 $O(1)$; 而当 $\lambda_{n}=n \lg n$ 时，(2.1) 式右方为无穷大, 这是不能令人满意的! 我们证得下面的

定理 2 设 $f \in \Lambda B V, \Lambda=\left\{\lambda_{n}\right\}$, 则 $f$ 的富里埃系数

$$
a_{n}(f)=\frac{1}{x} \int_{0}^{2 \pi} f(t) \begin{aligned}
& \cos n t \\
& \sin n t
\end{aligned} d t=O\left(1 / \sum_{v=1}^{n} \frac{1}{\lambda_{p}}\right)(n \rightarrow \infty) .
$$

定理 2 改进了定理 1, 因为

$$
\sum_{v=1}^{n} \frac{1}{\lambda_{p}} \geqslant \frac{n}{\lambda_{n}}
$$

另方面, 如 $f \in H B V$ ，则从 (2.2) 式可得

$$
\begin{aligned}
& a_{n}(f) \\
& b_{n}(f)
\end{aligned}=O(1 / \lg n) \quad(n \rightarrow \infty),
$$

而从 (2.1) 式只能得到 $a_{n}(f)=O(1)=b_{n}(f)$.

定理 3 设 $f \in A B V_{c}, A=\left\{\lambda_{n}\right\}$, 那么 (2.2) 式中的 $O$ 可以改成 $O$.

关于 $f$ 的积分连续模, 我们有

定理 4 设 $f \in \Lambda B V, \Lambda=\left\{\lambda_{n}\right\}$, 则 $f$ 的积分连续模

$$
\omega(\delta ; f)_{L_{1}}=\sup _{|t| i_{\delta}} \int_{0}^{2 \pi}|f(x+t)-f(x)| d x=O\left(1 / \sum_{v=1}^{\left[\frac{1}{\delta}\right]} \frac{1}{\lambda_{v}}\right) \quad(\delta \rightarrow 0) .
$$

定理 5 设 $f \in \Lambda B V_{c}, \Lambda=\left\{\lambda_{n}\right\}$ ，那么

$$
\omega(\delta ; f)_{L_{1}}=0\left(1 / \sum_{v=1}^{\left[\frac{1}{8}\right]} \frac{1}{\lambda_{p}}\right) \quad(\delta \rightarrow 0) .
$$

设 $\Lambda=\left\{\lambda_{i} ;, \Gamma=\left\{r_{\mathrm{s}}\right\}\right.$ ，那么函数类 $\Gamma B V \subset A B V$ 的充要条件是

$$
\sum_{k=1}^{n} \frac{1}{\lambda_{k}}=O\left(\sum_{k=1}^{n} \frac{1}{\gamma_{k}}\right) \quad(n \rightarrow \infty),
$$

而 $A B V=\Gamma B V$ 的充要条件是, 存在正数 $c_{1}$ 与 $c_{2}$, 使

$$
0<c_{1}<\sum_{k=1}^{n} \frac{1}{r_{k}} / \sum_{k=1}^{n} \frac{1}{\lambda_{k}}<c_{2}<\infty,
$$

这是文献 [2]的定理 3. 我们要问, 定理 2 中的估计式 (2.2) 式在阶的意义下是否精确? 就是 说, 当函数类 $A B V \subset \Gamma B V$ 且 $\Lambda B V \neq \Gamma B V$ 时，(2.2) 式对函数类 $\Gamma B V$ 是否仍成立? 
定理 6 设 $\Gamma B V \subset A B V$, 且 $\Lambda B V \neq \Gamma B V$, 那么存在函数 $f_{0} \in \Lambda B V$, 而 $f_{0}$ 的富里埃 系数

$$
b_{n}\left(f_{0}\right)=\frac{1}{x} \int_{0}^{2 \pi} f_{0}(t) \sin n t d t \neq O\left(1 / \sum_{\nu=1}^{n} \frac{1}{\lambda_{p}}\right) \quad(n \rightarrow \infty) .
$$

定理 6 说明,对于任意两个函数类 $A B V$ 与 $\Gamma B V$ ，假如 $\Gamma B V$ 是 $A B V$ 的真子集，那么就 整类而言, $A B V$ 中函数富里埃系数的阶估计式 (2.2) 式, 不能以较小的阶 $O\left(1 / \sum_{1}^{n} \frac{1}{r_{p}}\right)$ 来代 替.

\section{$\equiv$}

Waterman 在文献 [3] 中研究了 $A B V$ 与 $H B V$ 类中函数富里埃级数的绝对收敛问题. 他 的定理是

定理 B（文献 [3] 定理 4) 若 $f \in \Lambda B V, \Lambda=\left\{\lambda_{n}\right\}$, 那么级数

$$
\sum_{n=1}^{\infty} \lambda_{n}^{\frac{1}{2}} n^{-1} \omega^{\frac{1}{2}}\left(\frac{2 \pi}{n}\right)<\infty
$$

时, $f$ 的富里埃级数 $\odot[f]$ 绝对收玫; 假如 $f \in H B V$, 那么级数

$$
\sum_{n=1}^{\infty} n^{-\frac{1}{2}} \cos ^{\frac{1}{2}}\left(\frac{x}{n}\right)<\infty
$$

时, $\sigma[f]$ 绝对收敛,其中 $\omega(8)$ 是 $f$ 的连续模.

我们指出, 条件 (3.2) 式太苛刻, 因为 (3.2) 式是单调下降的正项级数, 它的收玫隐含

$$
n^{-\frac{1}{2} \omega^{\frac{1}{2}}}\left(\frac{\pi}{n}\right)=o\left(\frac{1}{n}\right) \quad(n \rightarrow \infty),
$$

即

$$
\omega\left(\frac{\pi}{n}\right)=\circ\left(\frac{1}{n}\right) .
$$

这样, 除常数之外, 不可能有函数满足条件 (3.2) 式. 其次, 假如 $f \in \Lambda B V, \Lambda=\left\{\lambda_{n}\right\}$ 而 $n=$ $o\left(\lambda_{*}\right)$, 那么条件 (3.1) 式更不合理,例如 $\lambda_{*}=n \lg n$ 时,条件 (3.1) 式只能对

$$
\omega\left(\frac{1}{n}\right)=o\left((n \lg n)^{-1}\right)
$$

的函数 $f$ 才有可能成立, 这当然是不合理的. 我们证得

定理 7 若 $f \in \Lambda B V, \Lambda=\left\{\lambda_{n}\right\}$, 那么级数

$$
\sum_{n=1}^{\infty}\left(\frac{\omega\left(\frac{1}{n} ; f\right)}{n \sum_{\nu=1}^{n} \frac{1}{\lambda_{p}}}\right)^{\frac{1}{2}}<\infty
$$

时, $\subseteq[f]$ 绝对收敛;若 $f \in H B V$, 级数

$$
\sum_{n=1}^{\infty}\left(\frac{\omega\left(\frac{1}{n} ; f\right)}{n \lg n}\right)^{\frac{1}{2}}<\infty
$$


时, $\Theta[f]$ 绝对收敛.

侵如 $A=\left\{\lambda_{n}\right\}, \lambda_{n}=n^{\beta}, 0<\beta<1 / 2$, 那么定理 7 尚可改进.

定国 8 若 $0 \leqslant \beta<1 / 2, \lambda_{n}=n^{\beta}(n=1,2, \cdots), A=\left\{\lambda_{n}\right\}$, 那么当 $f \in \operatorname{Lip} \alpha(\alpha>0)$ 时, $\theta[f]$ 绝对收敛.

从 Hardy-Littlewood 的例子(文献 [4] I 243. 页)

$$
f_{c}(x)=\sum_{n=1}^{\infty} \frac{e^{i_{1} 18 x}}{n} e^{\text {inx }} \in \operatorname{Lip} \frac{1}{2},
$$

可以知道, 定理 8 中的 $\alpha \leqslant 1 / 2$ 时, 条件 $f \in\left\{n^{\beta}\right\} B V(\beta>1 / 2)$ 对 $\Theta[f]$ 的绝对收敛起不了 什么作用.

\section{\% 文 蝴}

[1] Waterman, D., Studia Math., 57(1976), 33-45.

[2] - Proc. Amer. Math. Soc., 74(19.79), 119-123.

[ 3 ] - Studia Math., 44(1972), 107-117, 651.

[4] Zygmund, A., Trigonometric Series, Cambridge, 1959. 